ISSN 0103-5150

Fisioter. Mov., Curitiba, v. 24, n. 3, p. 419-428, jul./set. 2011 Licenciado sob uma Licença Creative Commons

\title{
Satisfação dos usuários dos serviços ambulatoriais de fisioterapia da rede pública
}

\author{
User satisfaction of clinical physical therapy in public health system
}

\author{
Andersom Ricardo Fréz ${ }^{[a]}$, Maria Inês Rubo de Souza Nobre ${ }^{[b]}$ \\ [a] Mestrado Profissional em Saúde, Interdisciplinaridade e Reabilitação, Universidade Estadual de Campinas (Unicamp), \\ Campinas, SP - Brasil, e-mail: andersom_frez@yahoo.com.br \\ [b] Professora Doutora do Centro de Estudos e Pesquisa em Reabilitação Prof. Dr. Gabriel Porto (Cepre), Faculdade de Ciências \\ Médicas (FCM), Universidade Estadual de Campinas (Unicamp), Campinas, SP - Brasil, e-mail: inesrsnobre@hotmail.com
}

\section{Resumo}

Introdução: Pesquisas de satisfação dos usuários são indispensáveis ao planejamento e à avaliação dos serviços de saúde, pois, quanto mais às políticas públicas de saúde estiverem justificadas pelo respeito às perspectivas e às necessidades dos usuários, mais estas políticas tenderão ao acerto. Objetivos: Caracterizar os usuários do serviço ambulatorial de fisioterapia da rede pública de saúde da cidade de Foz do Iguaçu, Paraná; identificar a conduta em relação à busca do atendimento fisioterapêutico; e descrever e quantificar a satisfação desses usuários após o atendimento. Materiais e métodos: Foi realizado um estudo transversal em um modelo epidemiológico descritivo, com aplicação de questionário validado e adequado socioculturalmente aos usuários desses serviços. Cento e três usuários se enquadraram nos critérios de inclusão e, destes, 99 questionários foram avaliados. Para análise dos dados aplicou-se o teste de Friedman para análise de variância não paramétrica das dimensões do questionário, complementado pelo pós-teste de comparação múltipla (post hoc de Dunn), analisando pares de dimensões. Resultados: A amostra caracterizou-se pelo predomínio de usuários do sexo feminino, com $2^{\circ}$ grau completo e renda familiar entre quatro e seis salários mínimos. A maioria foi atendida na especialidade ortopedia e traumatologia. Também relataram que já tiveram outras experiências com a fisioterapia e todos sabiam os seus diagnósticos. Em relação à satisfação, os usuários caracterizaram-na como ótima $(\mathrm{p}<0,0001)$ e observou-se analogia significativa entre as dimensões relação terapeuta/paciente e marcação de consulta $(\mathrm{p}<0,05)$ e entre a marcação de consulta 
e o retorno/recomendação da clínica $(\mathrm{p}<0,05)$; e uma relação extremamente significante entre a marcação de consulta e o acesso $(\mathrm{p}<0,01)$. Conclusão: As características da assistência fisioterapêuticas foram vistas como ótimas, com uma população satisfeita com os serviços que vem recebendo.

Palavras-chave: Avaliação em saúde. Satisfação do paciente. Fisioterapia. Saúde pública.

\section{Abstract}

Introduction: User satisfaction surveys are useful for the design and assessment of health services, because the more the public health policies are justified by respect for the perspectives and needs of users, most such policies tend to hit. Objectives: To characterize the users of clinical physical therapy service, in the public health care in the city of Foz do Iguaçu, Paraná; identify the conduct in relation to the search of physical therapy; describe and quantify user satisfaction after treatment. Materials and methods: A transversal descriptive study was realized, with an applications of a validated questionnaire, socially and cultural suitable to the users of those services. One hundred and three were fitted in the inclusion criteria and not in exclusion, and 99 questionnaires of these were analyzed. For data analysis we applied the Friedman test for analysis of variance nonparametric dimensions of the questionnaire, supplemented by post-test multiple comparison (Dunn's post hoc), analyzing pairs of dimensions. Results: The sample was characterized by female users' predominance, graduated in 2nd degree, with family rent between 4 and 6 minimal salaries. Orthopedic and traumatology were the main specialty attended. Also reported that they already had another experience with physical therapy and all of them knew their diagnoses. Relationed to satisfaction, the users characterized as great ( $p<$ $0,0001)$, and there was significant relationship between the dimension relationship therapist and patient and the appointment scheduling $(p<0,05)$ and between the appointment and the return/recommendation of the clinic $(p<0,05)$, and a highly significant relationship between the appointment scheduling and the access $(p<$ 0,01). Conclusion: Handling characteristics of physical therapy was seen as great, with a population satisfied with the services that has been receiving.

Keywords: Health evaluation. Patient satisfaction. Physical therapy. Public health.

\section{Introdução}

A fisioterapia foi regulamentada com o DecretoLei n. 938, de 13 de outubro de 1969 (1), e possui formação acadêmica (2) que destaca o fisioterapeuta como um profissional generalista, capaz de atuar em todos os níveis de atenção à saúde. Tendo, assim, ação sobre o princípio doutrinário do SUS: a integralidade. Contudo, frequentemente tem suas atividades reconhecidas na recuperação funcional de pessoas fisicamente lesadas, atuando, desse modo, em níveis de atenção secundária e terciária na saúde, influenciando, assim, a sua inserção na rede pública de saúde (3).

Atualmente, o SUS representa o maior empregador de trabalhadores em saúde e, de acordo com pesquisa realizada em 2003 pelo Ministério da Saúde (4), mais de $90 \%$ da população brasileira é usuária de alguma forma dos serviços de saúde do SUS. Entretanto, reconhece-se a baixa qualidade dos serviços oferecidos em termos de equipamentos e profissionais, a ausência de participação da população na formulação e gestão das políticas de saúde e a falta de mecanismos de acompanhamento, controle e avaliação dos serviços, pois esse processo avaliativo produz conhecimento sobre os sistemas e serviços de saúde com o objetivo de orientar o desenho de políticas e a melhoria do desempenho (5). Entretanto, no Brasil, o conceito de avaliação surgiu com a redemocratização na década de 1980 e com a implementação da Reforma Sanitária Brasileira, fortalecendo os movimentos de reivindicação por melhores condições no atendimento pelos serviços de saúde $(6,7)$.

Assim, as pesquisas de satisfação de usuários tornaram-se mais comuns na década de 1990 (8), tornando-se indispensáveis ao planejamento e à avaliação dos serviços de saúde (9), onde a satisfação pode ser entendida como o resultado do atendimento que foi prestado, pois manifesta a visão global dessa atividade e está impregnada pelos valores pessoais e 
sociais, bem como pelas experiências individuais. Dessa forma, a satisfação é multidimensional, sendo expressada de maneira diferente pelas pessoas, e uma mesma pessoa se manifestará diferentemente em tempos diferentes $(10,11)$, pondendo, ainda, estar satisfeita em vários aspectos da assistência e insatisfeita simultaneamente em outros (12).

Acredita-se, assim, que o planejamento e o redirecionamento das políticas públicas, particularmente daquelas relacionas à saúde, tenderão mais ao acerto quanto mais estiverem justificados pelo respeito às perspectivas e às necessidades dos usuários, as quais parecem passíveis de detecção e análise.

Dessa forma, a avaliação da satisfação dos usuários dos serviços de fisioterapia da rede pública de saúde de Foz do Iguaçu, PR, consistiu em um desafio, haja vista que eles se encontram em movimento de crescente expansão. Foz do Iguaçu está localizada em uma região de fronteira, oferecendo atendimento aos munícipes e aos brasileiros residentes nos países vizinhos. São 68 clínicas e consultórios, cadastrados no Crefito-8 (13), que prestam atendimento a essa população. Destas, cinco são credenciadas ao SUS. Além de três faculdades que também prestam atendimento fisioterapêutico.

Como atualmente a maior demanda é pelos serviços de recuperação funcional, que nunca foram submetidos a uma avaliação, o objetivo deste trabalho foi avaliar e analisar a satisfação dos usuários do SUS após atendimento fisioterapêutico oferecido no serviço ambulatorial da rede pública de saúde da cidade de Foz do Iguaçu, nas dimensões: relação terapeuta/paciente, ambiente físico, marcação de consulta, acesso e estrutura física. E também caracterizar esses usuários, por meio de variáveis sociodemográficas e econômicas, e identificar a forma de encaminhamento dos usuários para os serviços.

\section{Materiais e métodos}

A pesquisa caracterizou-se como um estudo transversal, baseado em um modelo epidemiológico descritivo, por meio de um questionário autoaplicável aos usuários do serviço de fisioterapia da rede pública de saúde da cidade de Foz do Iguaçu, PR. 0 estudo iniciou-se após a aprovação pelo Comitê de Ética em Pesquisa da Faculdade de Ciências Médicas da Universidade Estadual de Campinas (Parecer CEP $532 / 2008)$.
Consideraram-se como critérios de inclusão: usuário do serviço ambulatorial de fisioterapia da rede pública de saúde, maior de 18 anos; e de exclusão: usuários com dificuldade para comunicação escrita e verbal.

Foram identificadas cinco clínicas de fisioterapia que prestam atendimento aos usuários do SUS, sendo quatro particulares e uma do próprio município. Nesta, além dos atendimentos, os usuários agendam o início do tratamento e são encaminhados às demais clínicas. Após ciência e autorização da Secretária de Saúde, os responsáveis pelas clínicas foram abordados e todos autorizaram a coleta de dados. Assim, a população da pesquisa foram os usuários dos serviços de fisioterapia da rede pública de saúde, após alta do tratamento fisioterapêutico, no período de novembro a dezembro de 2008.

Foi aplicado o questionário: "Instrumento para aferir a satisfação do paciente com a assistência fisioterapêutica na rede pública", proposto e validado por Moreira et al. (5), sendo submetido a uma adequação sociocultural, com julgamentos de fisioterapeutas credenciados no sistema público de saúde e de usuários desses serviços.

Como o questionário foi desenvolvido no Rio Grande do Norte, mesmo com o procedimento de validação e adequação sociocultural, considerou-se prudente realizar previamente um estudo exploratório para verificar possíveis intercorrências na aplicação e na compreensão do questionário, avaliando a necessidade de uma adequação sociocultural para a população estudada.

Na segunda quinzena de outubro de 2008, 23 usuários fizeram parte do estudo exploratório. Todos assinaram o Termo de Consentimento Livre e Esclarecido. Não houve intercorrências na aplicação e na compreensão do questionário.

Assim, nos meses de novembro e dezembro de 2008, 103 usuários se enquadraram nos critérios de inclusão. Não houve recusa, todos assinaram o Termo de Consentimento Livre e Esclarecido e garantiu-se a confidencialidade e o anonimato das respostas. Quatro questionários foram excluídos por terem sido respondidos incorretamente/incompletamente, e os do estudo exploratório não foram analisados, sendo a amostra composta por 99 questionários.

Os usuários foram abordados e entrevistados por avaliadores externos - sem vínculo com as clínicas, e previamente treinados - logo após a alta do tratamento, garantindo, assim, que nenhum prejuízo 
ocorresse aos atendimentos, assim como nenhum ônus para estes, nem para as instituições credenciadas, pois as pesquisas realizadas na própria unidade de saúde, após a consulta, buscam resgatar a experiência concreta do usuário no serviço, evitando efeitos de perda de memória (9).

O questionário possuía duas partes: uma descritiva, com 11 questões para caracterizar a amostra; e outra objetiva, para avaliar a satisfação do usuário; e continha 32 questões, divididas em 6 dimensões: relação terapeuta/paciente (16 questões), marcação de consulta (2), ambiente físico (8), acesso (2) e estrutura física (2 questões). Todas apresentavam as mesmas cinco opções de respostas: péssimo, ruim, bom, ótimo e excelente. As duas últimas questões objetivas avaliavam se o usuário retornaria à clínica e se ele a recomendaria, também com cinco opções de respostas: nunca, não, talvez, sim, e com certeza.

Para analisar as respostas atribuiu-se valores a elas: péssimo/nunca valor 1 , ruim/não 2 , bom/talvez 3 , ótimo/sim 4 e excelente/com certeza 5; e atribuiu-se um descritor para a satisfação (Tabela 1).

Tabela 1 - Relação entre descritor do questionário, valor atribuído ao descritor e descritor da satisfação

\begin{tabular}{lcc}
\hline $\begin{array}{l}\text { Descritor do } \\
\text { questionário }\end{array}$ & $\begin{array}{c}\text { Valor atribuído } \\
\text { ao descritor }\end{array}$ & $\begin{array}{c}\text { Descritor da } \\
\text { satisfação }\end{array}$ \\
\hline Péssimo/nunca & 1 & Péssima/nunca \\
Ruim/não & 2 & Ruim/não \\
Bom/talvez & 3 & Boa/talvez \\
Ótimo/sim & 4 & Ótima/sim \\
Excelente/com certeza & 5 & Excelente/com certeza \\
\hline
\end{tabular}

Fonte: Dados da pesquisa.

A análise estatística descritiva incluiu mediana (Md), valores mínimos e máximos e distâncias interquartis (Q1, Md e Q3). Verificou-se a normalidade dos dados e, como não houve distribuição normal, utilizaram-se as Mds para caracterizar as dimensões e o teste de Friedman para análise de variância não paramétrica das dimensões, complementada pelo pós-teste de comparação múltipla (post hoc de Dunn), analisando pares de dimensões. Em todos os testes considerou-se o nível de significância de 5\% $(p<0,05)$. Utilizou-se o programa GraphPAd Instat.

\section{Resultados}

Dos 99 questionários, 38 (38,4\%) foram respondidos por homens e $61(61,6 \%)$ por mulheres. A idade média foi 42,1 anos (18-60 anos). Os sujeitos realizaram uma média de 68,6 atendimentos (10-200 atendimentos). Todos os usuários sabiam o seu diagnóstico clínico, 63,6\% foram atendidos por fisioterapeutas mulheres, $63,6 \%$ relataram que foi a primeira experiência na clínica que prestou os atendimentos e $58,5 \%$ que foi o primeiro contato com a fisioterapia.

Quanto às questões sociodemográficas e econômicas, pouco mais da metade $(50,5 \%)$ dos usuários referiram ter concluído o $2^{\circ}$ grau, aproximadamente $89 \%$ relataram ter renda familiar igual ou inferior a seis salários mínimos. Os principais motivos que conduziram os usuários aos serviços de fisioterapia foram os sintomas osteomusculares; e $66,7 \%$ dos usuários foram encaminhados/tiveram conhecimento da clínica que prestou atendimento pela unidade responsável pelos agendamentos e todos os casos respondidos como "outros" foram especificados pela proximidade da clínica da sua residência (Tabela 2).

Na parte objetiva, todas as dimensões apresentaram Md 4,0 (Tabela 3). Assim, quando convertido esse valor para o descritor a satisfação foi categorizada como ótima.

Quanto à distribuição das respostas, as dimensões marcação de consulta (MC) e ambiente físico e conveniência (AFC) apresentaram o maior intervalo nas respostas, de 1 a 5, e essas dimensões apresentaram os mesmos valores para Q1 e Q3: 3 e 5 respectivamente. Já as dimensões, relação terapeuta/ paciente (RTP), acesso (AC) e espaço físico (EF) apresentaram os mesmos valores para Q1 e Q3: 4 e 5 respectivamente, representando maior quantidade de respostas com os descritores ótimo e excelente. Já a dimensão retorno/recomendação da clínica (RRC) obteve o valor 4 para Q1 e Q3, tendo maior número de respostas com o descritor sim (Figura 1).

$\mathrm{Na}$ análise estatística das respostas, observou-se um resultado extremamente significante ( $p<0,0001)$. E no pós-teste observou-se uma relação significativa $(p<0,05)$ entre as dimensões relação terapeuta/ paciente e marcação de consulta e entre marcação de consulta e retorno/recomendação da clínica, e uma relação extremamente significante $(p<0,01)$ entre as dimensões marcação de consulta e acesso (Tabela 4). 
Tabela 2 - Perfil sociodemográfico e econômico dos usuários do serviço de fisioterapia oferecido na rede pública de saúde de Foz do Iguaçu, PR

\begin{tabular}{|c|c|c|}
\hline Dados avaliados & $f$ & $\%$ \\
\hline \multicolumn{3}{|l|}{ Escolaridade } \\
\hline 10 grau incompleto & 4 & 4 \\
\hline 1ํ grau completo & 14 & 14,1 \\
\hline $2^{\circ}$ grau incompleto & 19 & 19,2 \\
\hline $2^{0}$ grau completo & 50 & 50,5 \\
\hline Superior incompleto & 7 & 7,1 \\
\hline Superior completo & 5 & 5,1 \\
\hline \multicolumn{3}{|l|}{ Renda familiar (em salários mínimos) } \\
\hline 1 a 3 & 24 & 24,2 \\
\hline 4 a 6 & 64 & 64,6 \\
\hline 7 a 10 & 10 & 10,1 \\
\hline Mais de 10 & 1 & 1 \\
\hline \multicolumn{3}{|l|}{ Especialidade do atendimento } \\
\hline Ortopedia e traumatologia & 89 & 89,9 \\
\hline Neurologia & 3 & 3 \\
\hline Respiratória & 4 & 4 \\
\hline Uroginecologia & 2 & 2 \\
\hline Mastologia & 1 & 1 \\
\hline \multicolumn{3}{|l|}{ Forma de encaminhamento } \\
\hline Unidade responsável pelo agendamento & 66 & 66,7 \\
\hline Proximidade à residência & 19 & 19,2 \\
\hline Indicação do médico & 2 & 2 \\
\hline Indicação de paciente anterior & 12 & 12,1 \\
\hline
\end{tabular}

Fonte: Dados da pesquisa.

\section{Discussão}

A pesquisa realizada despertou interesse nos gestores responsáveis pelos serviços de fisioterapia, por ser uma avaliação nunca realizada. Esta pesquisa também demonstrou sua importância tendo em vista que na literatura científica encontra-se um número reduzido de estudos referentes à satisfação do usuário de serviços de fisioterapia oferecidos pelo SUS e por instituições privadas.

Inicialmente, acreditava-se na insatisfação dos usuários em relação aos serviços oferecidos. Contudo, em todas as questões e dimensões os usuários ca-
Tabela 3 - Medianas e frequências dos valores atribuídos às respostas das dimensões e seu respectivo descritor

\begin{tabular}{lccc}
\hline Dimensão & Md & $\boldsymbol{f}$ & $\begin{array}{c}\text { Descritor da } \\
\text { satisfação }\end{array}$ \\
\hline Relação terapeuta/paciente & 4 & 48 & Ótima \\
Marcação de consulta & 4 & 39 & Ótima \\
Ambiente físico e conveniência & 4 & 43 & Ótima \\
Acesso & 4 & 49 & Ótima \\
Estrutura física & 4 & 46 & Ótima \\
Retorno/recomendação & 4 & 76 & Sim \\
da clínica & & & retornaria/ \\
Todo questionário & & & recondaria) \\
\hline
\end{tabular}

Legenda: $\mathrm{Md}=$ mediana.

Fonte: Dados da pesquisa.

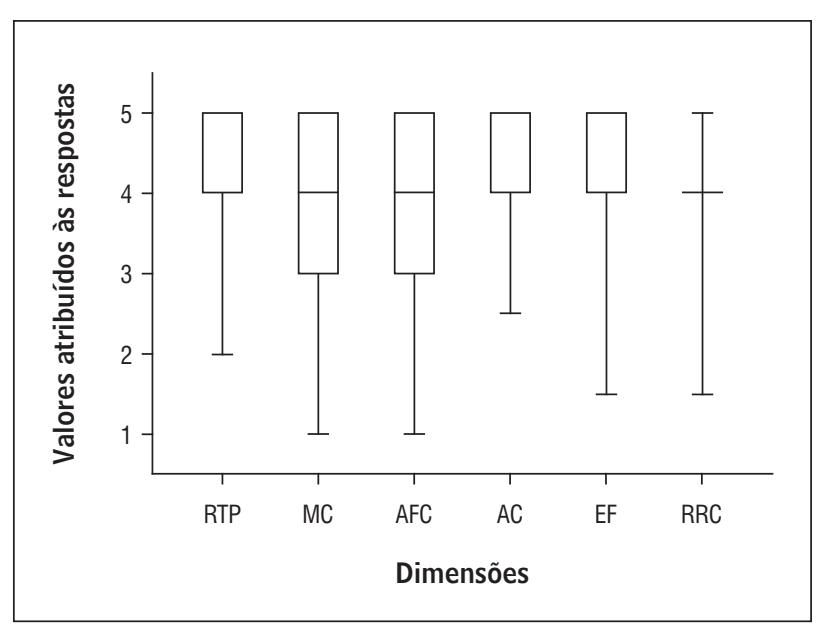

Figura 1 - Distribuição das respostas, valores mínimos, $1^{0}$ quartil, mediana, 30 quartil e valores máximos

Fonte: Dados da pesquisa.

racterizaram a satisfação com o serviço como ótima. Salienta-se que na análise individual existem usuários insatisfeitos, mas não quando analisada a coletividade. Em estudos sobre satisfação dos usuários com serviços de fisioterapia, Pereira (14) observou em sua amostra mais de $76 \%$ de usuários satisfeitos com o serviço e Magalhães (15), 82,6\%.

Segundo Esperidião e Trad (9), a maioria dos estudos, entre abordagem qualitativa e quantitativa, traz como resultados altas taxas de satisfação, sendo esse efeito reportado mesmo quando as expectativas 
Tabela 4 - Relação entre as dimensões

\begin{tabular}{ll}
\hline Comparação & Valor de p \\
\hline Relação terapeuta/paciente $\times$ Marcação de consulta & $p<0,05^{\star}$ \\
Relação terapeuta/paciente $\times$ Ambiente físico e conveniência & $p>0,05$ \\
Relação terapeuta/paciente $\times$ Acesso & $p>0,05$ \\
Relação terapeuta/paciente $\times$ Estrutura física & $p>0,05$ \\
Relação terapeuta/paciente $\times$ Retorno/recomendação da clínica & $p>0,05$ \\
Marcação de consulta $\times$ Ambiente físico e conveniência & $p>0,05$ \\
Marcação de consulta $\times$ Acesso & $p<0,01^{\star *}$ \\
Marcação de consulta $\times$ Estrutura física & $p>0,05$ \\
Marcação de consulta $\times$ Retorno/recomendação da clínica & $p<0,05^{\star}$ \\
Ambiente físico e conveniência $\times$ Acesso & $p>0,05$ \\
Ambiente físico e conveniência $\times$ Estrutura física & $p>0,05$ \\
Ambiente físico e conveniência $\times$ Retorno/recomendação da clínica & $p>0,05$ \\
Acesso $\times$ Estrutura física & $p>0,05$ \\
Acesso $\times$ Retorno/recomendação da clínica & $p>0,05$ \\
Estrutura física $\times$ Retorno/recomendação da clínica & $p>0,05$ \\
\hline
\end{tabular}

Legenda: * $=$ significante; ${ }^{* *}=$ extremamente significante.

Fonte: Dados da pesquisa.

sobre os serviços são negativas. Esses autores questionam, ainda, se é provável que os profissionais de saúde sempre realizem um "cuidado perfeito". Moreira et al. (5) citaram o fato como falta de visão crítica, justificada pela gratuidade do serviço prestado, além de ser uma tentativa de agradar para acentuar os benefícios. Deve-se atentar para uma tendência já comprovada por parte dos usuários de classes sociais menos favorecidas: a de avaliar positivamente os serviços que lhes são prestados. Vaitsman e Andrade (7) citaram que o usuário pode ter aprendido a diminuir suas expectativas quanto aos serviços e enfatizam que uma boa avaliação pode ser fruto de uma baixa capacidade crítica dos usuários. Williams (16) relatou que uma avaliação positiva não quer dizer necessariamente que houve avaliação crítica, podendo expressar ausência de opinião e/ou a aceitação do paternalismo dos profissionais da saúde. Para Esperidião e Trad (9), nos países em desenvolvimento, como o Brasil, espera-se que os usuários manifestem mais insatisfação com os serviços que lhes são oferecidos. Contudo, os usuários apresentam-se em uma atitude passiva em face do serviço (17).

Nos serviços públicos, o poder de pressão dos usuários é baixo, pois não há necessidade de captação de clientela, como no setor privado, e as avaliações deveriam representar um importante meio, atuando como instrumento de voz dos usuários (9). Bernhart (18) relatou que no setor público a avaliação feita a partir dos usuários envolve uma interação mais complexa de elementos, incluindo os que influenciam as percepções sobre os serviços públicos, entre eles o sentimento de gratidão. A dificuldade usual de conseguir atendimento resulta em baixa expectativa, e o simples fato de ser atendido já pode produzir satisfação, pois as pessoas não esperam muito das instituições públicas (7). Assim, os usuários evitariam criticar os serviços em virtude desse viés, tanto pelo medo de perder o acesso quanto à relação de dependência aos profissionais de saúde.

Apesar dos altos percentuais definidos como bom e ótimo não se pode considerar que as condutas fisioterapêuticas foram seguidas adequadamente, pois o questionário avaliava a satisfação do usuário com o cuidado prestado, e não o resultado alcançado ou os procedimentos aplicados. Mendonça e Guerra (19) afirmaram que para julgar o resultado da assistência prestada se faz necessário uma avaliação que inclua capacidade funcional e qualidade de vida. 
Para mensurar a satisfação, a literatura traz vários questionários. Contudo, quando se trata da fisioterapia existem características que podem influenciar a satisfação do paciente. A intervenção, frequentemente, demanda muito mais tempo que uma visita clínica rotineira, além de envolver contato físico com a terapia, normalmente, requerer a participação do paciente (19). Essas características citadas não foram consideradas/observadas na presente pesquisa, porém se observou usuários submetidos a até 200 atendimentos. Segundo Moreira et al. (5), é possível que ocorram interferências nas respostas dos usuários submetidos a intervenções de longa duração, necessárias ao tratamento de disfunções crônicas.

Outro viés para estudos transversais (20) é o ocorrido na seleção dos sujeitos. No sentido de que aqueles que não responderam o questionário possam ter diferentes opiniões em relação aos que participaram do estudo. No entanto, no presente estudo a população foi composta por todos os usuários que receberam alta do serviço. E o fato do questionário ter sido autorrespondido também pode ser considerado como fato positivo, pois garante o sigilo das informações e minimiza o viés da informação.

Mendonça et al. (21) complementaram que, em cada contexto cultural, a satisfação do usuário com a assistência recebida é influenciada por características sociodemográficas dos usuários, em especial no tocante ao sexo do paciente, renda familiar e nível educacional.

Assim, quanto à caracterização dos usuários, observou-se um predomínio do sexo feminino $(61,6 \%)$, que também é enfatizado em outros estudos sobre serviços de fisioterapia, como na pesquisa de Pereira (14), na qual observaram uma prevalência de 59,5\% de mulheres, e $62,5 \%$ no trabalho de Machado e Nogueira (8). Estes (8) relataram que a mulher tem maior facilidade para desenvolver complicações osteomusculares, pois, além dos afazeres domésticos, muitas são responsáveis pelo sustento da família. Nesse sentido, esta pesquisa reforça outros estudos, que registraram o predomínio do sexo feminino na procura dos serviços de saúde. Pinheiro e Travassos (22) identificaram maior número de mulheres dentre os frequentadores de serviços de saúde na cidade do Rio de Janeiro. 0 mesmo se dá no trabalho de Rosso e Silva (23), na análise sobre a qualidade do atendimento em unidades básicas de saúde (UBS) em Tubarão, SC, com 87,1\% de mulheres. Predomínio também observado por Corrêa (24), que avaliou os moradores próximos a UBS.

No estudo de prevalência de distúrbios osteomusculares em bancários, Brandão et al. (20) observaram um predomínio de mulheres que referiam dor osteomuscular. No processo de tradução e validação de um questionário de sintomatologia dolorosa osteomuscular, Pinheiro et al. (25) também observaram a prevalência desses sintomas em mulheres. No presente estudo, $90 \%$ dos usuários foram atendidos na especialidade ortopedia/traumatologia, destes $60,7 \%$ eram do sexo feminino. Gomes et al. (26) concluíram, em sua pesquisa qualitativa, que a baixa procura dos homens pelos serviços de saúde se deve à dificuldade na adoção de práticas de autocuidado, pois procurar o serviço de saúde poderia associá-lo à fraqueza, medo e insegurança; portanto, poderia aproximá-lo das representações do universo feminino. Os mesmos autores também observaram que a ausência dos homens nos serviços de saúde seria o medo da descoberta de uma doença grave; e a organização desses serviços foi considerada pouco apta em absorver a demanda apresentada pelos homens, pois sua organização não estimula o acesso.

A idade média observada foi de 42,1 anos (18-60 anos). Oliveira et al. (27) encontram resultados semelhantes em relação à média de idade dos pacientes (46,5 anos), em estudo sobre as características e a satisfação do ambulatório de oftalmologia de um hospital universitário. Já Corrêa (24) observou que a população que mais procura atendimento nas UBS está na faixa de 21 a 45 anos.

Em relação à escolaridade, observou-se um predomínio de usuários com $2^{\circ}$ grau completo $(50,5 \%)$, frequência diferente do estudo de Machado e Nogueira (8), no qual observaram um predomínio de analfabetos $(31,1 \%)$. Enquanto Corrêa (24) encontrou um predomínio entre usuários das UBS de 1ํo grau incompleto; e Machado (28) verificou que entre os usuários da rede pública impera o analfabetismo. Destaca-se que os estudos foram realizados em regiões distintas. Os trabalhos de Machado e Nogueira (8) e Machado (28) foram realizados em Teresina, PI, enquanto o de Corrêa (24) foi realizado em Campinas, SP.

A maioria dos usuários $(64,6 \%)$ referiu ter renda familiar entre quatro e seis salários mínimos. Já na amostra de Machado e Nogueira (8), apenas $4,5 \%$ referiram ter essa renda, a maioria $(64,6 \%)$ 
referiu-a entre um e três salários. Enquanto no trabalho de Rosso e Silva (23) os autores constataram que os usuários de UBS têm renda inferior a três salários. E Machado (28) verificou que entre os usuários da rede pública existe o predomínio de profissionais autônomos, sem vínculo empregatício e, portanto, com renda entre um e três salários.

Quanto à localização geográfica, aproximadamente $20 \%$ relataram escolher a clínica prestadora dos atendimentos graças à proximidade de sua residência. Enquanto no estudo de Corrêa (24) 12\% referiram que utilizavam determinada UBS em razão da proximidade de sua residência.

Moreira et al. (5), Mendonça e Guerra (19) e Mendonça (29) relataram que o domínio que apresenta a maior correlação com a satisfação do usuário é a interação terapeuta/paciente. Eles salientam que a comunicação entre o terapeuta e o paciente vem sendo discutida como um dos mais importantes aspectos da satisfação. Contudo, no presente estudo observou-se uma equivalência nas dimensões. Ressalta-se que na dimensão acesso, principalmente no tocante ao acesso de pessoas com deficiência física, foi observado o menor intervalo entre o menor e o maior valor das questões, além de ter o maior número de respostas "excelente". Contrariando, assim, os resultados encontrados por Moreira et al. (5), no qual concluem que dimensões referentes à acessibilidade estão menos relacionadas ao construto satisfação.

Corroborando com outros estudos sobre avaliação do serviço de fisioterapia $(5,8)$, as dimensões que os usuários referiram à menor satisfação estavam relacionadas com a dificuldade para marcar e iniciar o tratamento. Tal fato poderia ser justificado pela quantidade limitada de atendimentos, porém, seguindo as diretrizes da Portaria n. 1101/02 (30), Foz do Iguaçu presta mais atendimentos que o preconizado. No entanto, existe um fator que dificulta $o$ agendamento do primeiro e dos subsequentes atendimentos: a não informatização do processo. Assim, o usuário deve se encaminhar, mensalmente, até a clínica de fisioterapia do município para agendar seus atendimentos. Sugere-se aos gestores que o atual modelo de agendamentos seja alterado: a informatização do sistema facilitaria o usuário, assim ele poderia realizar esses procedimentos na UBS próxima à sua residência, ou na própria unidade que o encaminhou para tratamento fisioterapêutico.

Em relação aos domínios estrutura física, recepção, limpeza dos ambientes e conforto, a maioria dos usuários classificou-os como ótimo. Também a confiança nos resultados dos tratamentos é comprovada diante da predisposição dos usuários em indicarem o serviço a terceiros. Confiança também observada entre os usuários de UBS, nas quais a maioria dos usuários relatou que consumiria os serviços oferecidos pelas unidades (24).

Partiu-se do pressuposto de que se tinha como resultados esperados uma população insatisfeita com os serviços de fisioterapia oferecidos na rede pública de saúde em Foz do Iguaçu, PR. Contudo, o estudo mostrou uma população satisfeita com os serviços que vem recebendo. E apesar de diversos autores afirmarem que esse fato se deve à baixa capacidade crítica $\mathrm{e}$ avaliativa dos usuários e o não conhecimento de seus plenos direitos, na presente pesquisa observou-se uma situação particular, na qual a maioria de usuários referiu ter o $2^{\circ}$ grau completo e com remuneração entre quatro e seis salários mínimos. Sendo uma população diferente da maioria dos usuários dos serviços públicos de saúde, com renda familiar acima da média da população brasileira e com grau de instrução elevado e, supõe-se, com um nível de exigência mais acurado e, dessa forma, capaz de avaliar criticamente o serviço. Assim, questiona-se se realmente existe essa baixa capacidade em avaliar serviços e programas de saúde, se os usuários superdimensionam os serviços ou, ainda, se essa baixa expectativa dos usuários em relação aos serviços de saúde deva ser considerada. Lança-se, então, a hipótese: será que os serviços já atendem à expectativa dos usuários e, dessa forma, sua satisfação com o esse serviço se expressa como ótima?

\section{Conclusões}

Os usuários do serviço de fisioterapia da rede pública de saúde de Foz do Iguaçu, PR, caracterizamse por um predomínio de pessoas do sexo feminino, tendo metade dos usuários o $2^{\circ}$ grau completo, e grande parcela deles com renda familiar entre quatro e seis salários mínimos. A maioria foi atendida na especialidade ortopedia e traumatologia e também relatou que já teve outras experiências com a fisioterapia. Todos sabiam os seus diagnósticos. Para a busca do serviço, a maioria foi encaminhada às clínicas credenciadas de acordo com a orientação da unidade responsável pelos agendamentos.

Na visão dos usuários, todas as dimensões tiveram a satisfação caracterizada como ótima. Além de 
afirmarem que retornariam e recomendariam a clínica que prestou os atendimentos fisioterapêuticos. Assim, as características da assistência fisioterapêuticas foram vistas como ótimas, com uma população satisfeita com os serviços que vem recebendo. Contudo, relação significativa foi observada entre a dimensão marcação de consultas, quando analisada com a relação terapeuta/paciente, acesso e retorno/recomendação da unidade.

\section{Referências}

1. Brasil. Decreto-Lei n. 938, de 13 de outubro de 1969. Dispõe sobre a criação das profissões de fisioterapeuta e terapeuta ocupacional. Diário Oficial da República Federativa do Brasil, Brasília, DF, 14 out. 1969. [acesso em 15 dez. 2008]. Disponível em: http:// www.coffito.org.br / legislacao2.asp?id=11

2. Brasil. Ministério da Educação. Resolução CNE/ CES 4, de 19 de fevereiro de 2002. Diretrizes Curriculares Nacionais do Curso de Graduação em Fisioterapia. [acesso em 20 out. 2009]. Disponível em: http://portal.mec.gov.br/cne/arquivos/pdf/CES04 2002.pdf

3. Ribeiro KSQS. A atuação da fisioterapia na atenção primária à saúde: reflexões a partir de uma experiência universitária. Fisioter Brasil. 2002;3(5):311-8.

4. Brasil. Ministério da Saúde. Ministério da Saúde lança QualiSUS em hospitais do Rio de Janeiro. [acesso em 15 ago. 2009]. Disponível em: http://portal.saude. gov.br/saude

5. Moreira FM, Borba JAM, Mendonça KMPP. Instrumento para aferir a satisfação do paciente com a assistência fisioterapêutica na rede pública de saúde. Fisioter Pesq. 2007;14(3):37-43.

6. Uchimura KY, Bosi MLM. Qualidade e subjetividade na avaliação de programas e serviços em saúde. Cad Saúde Pública. 2002;18(6):1561-9.

7. Vaitsman J, Andrade GRB. Satisfação e responsividade: formas de medir a qualidade e a humanização da assistência à saúde. Ciênc Saúde Coletiva. 2005; 10(3):599-613.

8. Machado NP, Nogueira LT. Avaliação da satisfação dos usuários de serviços de fisioterapia. Rev Bras Fisioter. 2008;12(5):401-8.
9. Esperidião M, Trad LAB. Avaliação de satisfação de usuários. Ciênc Saúde Coletiva. 2005;10(Sup 1): 303-12.

10. Hall JA, Dornan MC. Patient sociodemographic characteristics as predictors of satisfaction with medical care: a meta-analyis. Soc Sci Med. 1990;30(7):811-8.

11. Harpole LH, Orav EJ, Hickey M, Posther KE, Brennan TA. Patient satisfaction in the ambulatory setting. J Gen Intern Med. 1996;11(7):431-4.

12. Beattie PF, Pinto MB, Nelson MK, Nelson R. Patient satisfaction with outpatient physical therapy: instrument validation. Phys Ther. 2002;82(6):557-65.

13. Conselho Regional de Fisioterapia e Terapia Ocupacional da 8a Região. Dados estatísticos. Posição dos núcleos para fiscalização. Dados do $2^{\circ}$ semestre de 2009. [acesso em 15 dez. 2009]. Disponível em: http:// www.crefito8.org.br/dados_det.php?cod=16

14. Pereira FWA. Avaliação do serviço de fisioterapia de média complexidade ambulatorial do SUS no município de Sobral - CE. [monografia]. Sobral: Universidade Vale do Acaraú; 2004.

15. Magalhães MS. Avaliação da assistência fisioterapêutica sob a óptica do usuário. [dissertação]. Fortaleza (CE): Universidade Federal do Ceará; 2002.

16. Williams B. Patient satisfaction: a valid concept? Social Science and Medicine. 1994;38(4):509-16.

17. Esperidião MA, Trad LAB. Avaliação de satisfação de usuários: considerações teórico-conceituais. Cad Saúde Pública. 2006;22(6):1267-76.

18. Bernhart MH, Wiadnyana IGP, Wihardjo H, Pohan I. Patient satisfaction in developing countries. Social Science and Medicine. 1999;48:989-96.

19. Mendonça KMPP, Guerra RO. Desenvolvimento e validação de um instrumento de medida da satisfação do paciente com a fisioterapia. Rev Bras Fisioter. 2007;11(5):369-75.

20. Brandão AG, Horta BL, Tomasi E. Sintomas de distúrbios osteomusculares em bancários de Pelotas e região: prevalência e fatores associados. Rev Bras Epidemiol. 2005;8(3):295-305.

21. Mendonça KMPP, Guerra RO, Diógenes TPM, Lima TMFS. Influência das características sócio-demográficas na satisfação do paciente com tratamento fisioterapêutico. Fisioter Mov. 2006;9(3):83-6. 
22. Pinheiro RS, Travassos C. Inequality in health care use by the elderly in three districts in the city of Rio de Janeiro. Cad Saúde Pública. 1999;15(3):487-96.

23. Rosso JA, Silva RM. Avaliação da qualidade do atendimento em unidades primárias de saúde: comparação de estruturas com e sem a presença de acadêmicos de medicina. Arq Catarinenses Med. 2006;35(2):47-55.

24. Corrêa, CRS. Avaliação das unidades básicas de saúde segundo os consumidores: a associação entre dados de produção das UBS da Campinas e as avaliações prévia e posterior dos moradores das áreas de cobertura dessas unidades. [tese]. Campinas: Universidade Estadual de Campinas; 1998.

25. Pinheiro F, Trócoli B, Carvalho C. Validação do questionário nórdico de sintomas osteomusculares como medida de morbidade. Rev Saúde Pública. 2002;36(3): 307-12.

26. Gomes R, Nascimento EF, Araújo FC. Por que os homens buscam menos os serviços de saúde do que as mulheres? As explicações de homens com baixa escolaridade e homens com ensino superior. Cad Saúde Pública. 2007;23(3):565-774.

27. Oliveira DF, Arieta CEL, Temporini ER, Kara-José N. Quality of health care: patient satisfaction in a university hospital. Arq Bras Oftalmol. 2006;69(5):731-6.
28. Machado NP. Avaliação da satisfação dos usuários de serviços de fisioterapia em Teresina - Piauí. [dissertação]. Teresina: Universidade Federal do Piauí; 2008.

29. Mendonça KMPP. Satisfação do paciente com a fisioterapia: tradução, adequação cultural e validação de um instrumento de medida. [dissertação]. Natal: Universidade Federal do Rio Grande do Norte; 2004.

30. Brasil. Ministério da Saúde. Portaria n. 1101, de 12 de junho de 2002. Brasília, DF; 2002. [acesso em 15 ago. 2009]. Disponível em: http://www.portal.saude. gov.br/portal/arquivos/pdf/portaria\%20gm $\% 20$ 1101\%2002.pdf

Recebido: $10 / 12 / 2010$

Received: $12 / 10 / 2010$

Aprovado: $14 / 04 / 2011$

Approved: 04/14/2011 